\title{
Personalized diet oriented by artificial intelligence and ethnic foods
}

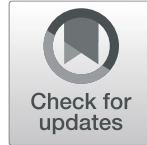

Dae Young Kwon ${ }^{1,2}$

\begin{abstract}
In the future, societies will endeavor to move beyond the framework of the industrial economy and the world will enter a new era marked by super-aged populations, artificial intelligence (Al), and the lifestyle economy. It is predicted that humanity will shift away from the industrial economy to a lifestyle economy where the main focus is human happiness. Human thought and behavior is influenced by emotions and cannot be explained entirely through rational decision-making. Accordingly, Al and other super connection technologies that drive big data and deep learning will facilitate the introduction of personalized and decentralized services, which represent a shift away from the era of mass production, centralization and automation. This means that more attention will be paid to designing unique lifestyles instead of production. In the future, super-aged societies will no longer be steeped in individualism, and become more people-centric. In such societies, obtaining an accurate understanding of people's noneun style (other activities style beyond working as leisure, play, and others) and dietary style to deliver personalized foods conducive to healthy eating and healthy lifestyles will be of great importance. Personalized diets will be developed based on structured data drawn from human genes, which do not change. However, unstructured data about epigenetics and microbiomes, which can be influenced by lifestyle and diet, will be important for designing personalized foods and lifestyles. Diversity in foods is the most important factor in generating this unstructured data. Because the responsibility for maintaining a healthy diet and lifestyle falls on individuals, developing personalized food and lifestyle products is an important step towards reaching the centenarian era. Developing personalized diets requires technologies such as super-connectivity, Al, and the Internet of humans (loHuman) rather the internet of things (IoT), but researching these technologies is not a job for the food industry. Technologies developed in other fields can be used in food science research, and these technologies fall under the umbrella of "foodomics." Such technologies include genetic analysis, whole genome sequence analysis, nutrigenomics, metabolomics, nutrigenetics, nutriepigenetics, microbiome technology, sensomics, and culturomics, as well as "sikdanomics." Korea has a lot of potential when it comes to personalized foods and diets. Because Korea has a long agricultural history, the country has a wide range of natural foods, and many ways to prepare and eat these foods. This means that Korean foods, in particular traditional Korean foods, are well positioned to meet the demand for diversity in personalized foods. In a super-aged era characterized by Al, Korea has a good chance of becoming a country where people can live to 100 in good health and also has the potential to be a global leader in personalized diets. To achieve this, food scientists and medical professionals need to work together to convince the Korean government to lead the industry in the right direction.
\end{abstract}

Keywords: Fourth industrial revolution, Artificial intelligence (Al), Personalized diet, Foodomics, Epigenetics

\footnotetext{
Correspondence: dykwon@kfri.re.kr

${ }^{1}$ Korea Food Research Institute, 245, Agribio Rd., Iso-myun, Wanju-kun,

Chollabuk-do 55365, Republic of Korea

${ }^{2}$ Korea Academy of Science and Technology, 42, Dolmaro, Bundang-ku,

Songnam, Kyongki-do, Republic of Korea
}

(c) The Author(s). 2020 Open Access This article is distributed under the terms of the Creative Commons Attribution 4.0 International License (http://creativecommons.org/licenses/by/4.0/), which permits unrestricted use, distribution, and reproduction in any medium, provided you give appropriate credit to the original author(s) and the source, provide a link to the Creative Commons license, and indicate if changes were made. The Creative Commons Public Domain Dedication waiver (http://creativecommons.org/publicdomain/zero/1.0/) applies to the data made available in this article, unless otherwise stated. 


\section{Introduction}

Many people like to discuss and make predictions about future societies. Some talk about population problems, while others discuss economic issues or what our lives will look like in the future. During the 1960s and 1970s, when industrialization was in full force, a lot of futurologists believed that the food industry would become dominated by processed foods, as this was the most efficient way to obtain the caloric intake necessary to fuel productive work. Some even predicted that people would only need to eat a single capsule per day in the future, and a number of food scientists openly stated that cultures like Korea where foods were difficult to prepare, took a long time to eat, and produced a lot of dishes would end-up disappearing. Following this logic, many advocated that all foods should be processed and made more convenient, and the tone of these policies still has a lasting impact on development in the Korean food industry today.

However, these predictions proved to be incorrect. This is because food scientists focused their attention only on food's primary function of providing calories, while ignoring other aspects of food production. Eating is not just about nutrition. It is also about enjoying the food's taste, finding joy in the act of eating, and interacting with others. However, food scientists failed to take this into account, viewing time spent eating as an obstacle to industrialization that needed to be removed.

Nowadays, the world takes great interest in the history, culture, and health benefits of Korean food, and many scientists and futurologists believe Korean food has great potential to grow further in the global food and food culture market. With growing interest in gastronomy, alongside the culture of finding joy in preparing and eating food together with others, the rise of "slow food," including foods which take a long time to prepare (and were the enemy of efficiency-minded food scientists) has boosted the value of Korean food.

Although it is difficult to predict the future, it is possible to ascertain some characteristics of what future societies might look like. The first is that society will continue to transition away from the industrial paradigm which is marked by competition through production and move towards "post-industrial logic" which is more people-centric and in tune with nature. However, human activities and lifestyles are also likely to drastically change as a result of technological advancement, especially AI (artificial intelligence) [1]. Second, advancement in medicine and biotechnology will extend the human lifespan, leading to super-aged societies. This means humanity will transition from an industrial economy to a lifestyle economy that is focused on human happiness. The industrial economy will take a backseat within the broader framework of the lifestyle economy. The third industrial revolution was marked by great advances in productivity that was made possible through high-speed calculations. However, the development of core technologies such as AI will help humanity move past this mindset to reach the 4th IR, where the introduction of machines that are able to "think" using deep learning technology capable of analyzing human cognition and behavior will lead to a greater focus on individual lifestyles instead of production.

In the book "The Fourth Industrial Revolution (4th IR)," Executive Chairman Klaus Schwab of World Economic Forum (WEF) advocates for changes to our economic systems and social structures based on the AI revolution brought about by convergence between digital and bio-technology [2]. Nations around the world, including the USA, Japan, Germany, and China, have realized that they need to prepare for the Fourth Industrial Revolution, and laid out strategies to this end. This is a highly sensitive issue in Korea. The Fourth Industrial Revolution is the latest in a line of buzzwords including "going green," "sustainable growth," and the "creative economy." In fact, Korea accounts for up to $97 \%$ of online searches worldwide for the Fourth Industrial Revolution. The Korean government believes that Korea will fall behind in global competition if it fails to adapt to the Fourth Industrial Revolution, and it appears that relevance to the 4th IR and the potential to develop future economic growth engines are treated as important criteria for evaluating the activities of all government departments. In fact, 4th IR has even become a hot topic in religious circles.

However, behind this enthusiasm lies the fact that a misguided understanding of the 4th IR can lead to serious consequences. One example is viewing the 4th IR through the lens of the production economy, believing that it merely represents an extension of the automation, convenience, and efficiency that marked the Third Industrial Revolution [3]. This is rooted in a false understanding of the nature of the 4th IR, and the Korean government continues to operate on the understanding that the Korean economy is purely an industrial economy. Although there is a lot of talk about the development of autonomous vehicles through AI technology, the 4th IR is about more than just producing autonomous vehicles to beat the competition. It is about the social and lifestyle changes that will be brought about by technology such as autonomous vehicles. In the same vein, the discourse about the 4th IR in the agricultural sector is mainly focused on 3D printing, smart farms, and smart logistics only in terms of profits of company than the happiness of human. This obsession with production is merely an extension of the Third Industrial Revolution (3rd Industrial Revolution) and does not truly represent the 4th IR. In discussions about the 
essence of the 4th IR, very few people are talking about the issues of social structures and social values or how the agricultural sector can produce food which helps people live happy and healthy lives. An example of real 4th IR technology is the use of 3D printing to produce customized human organs that function as nerves, veins, or muscles based on big data analysis. Similarly, instead of focusing on technological factors such as facilities, the production environment, and automation, discussions about smart farms should center around the use of block-chain technology to produce a diverse range of personalized foods through processes such as "hormesis" that can fulfill the biological and cultural needs of consumers [4]. This means that food industry should be considered highly relevant to the 4th IR. It appears that the food industry was passed over because those working in the industry do not have a complete understanding of the 4th IR and failed to convince the government of their industry's importance. However, before placing the blame on those in the industry, it is important for food scientists including myself to admit their share of responsibility.

It is crucial that those in the food industry have an accurate understanding of the 4th IR and use this as a foundation for engaging in discussions with the government. At present, claiming that the food industry has the greatest potential of all industries in the 4th IR is likely to be met with quizzical stares or even scorn. This is the reality we live in. However, because the 4th IR represents a great change in the structure of human lifestyles, the food industry is likely to be at the forefront of the revolution since it accounts for a large proportion of the leisure industry. Due to advancement in AI and biotechnology [5], diet and eating habits will continue to play an ever more important role in human health, so accurate analysis of consumer demands for health, taste, and wellbeing will form an important part of providing personalized food and lifestyle products. This will serve as the foundation for sustaining happy and healthy lives, and it is the 4th IR that will enable people to live such lives.

For example, when providing personalized food product services, companies may be able to obtain genetic information about people through deep learning to make accurate predictions, then provide dietary guidelines to individuals to maximize their health and happiness. Providing personalized food and lifestyle products based on an accurate understanding of each individual's leisure and dietary style will be particularly important in the super-aged, personalized, people-centric societies of the future. Personalized diets will be developed based on structured data drawn from human genes, which do not change. However, unstructured data about epigenetics and microbiomes, which can be influenced by lifestyle and diet, will be an important form of big data for designing personalized foods. This is why it is important to understand lifestyle patterns as well as structured data about personalized diets, unstructured genetic data about individuals, epigenetic data, and microbiome data.

Super-connectivity, AI, and the IoT are the hardware technologies that lie at the heart of the 4th IR, while interactive data systems (platforms), technology to recognize lifestyles and lifestyle patterns, biotechnology, machine learning, and deep learning make up the core software. Big data is also part of this. The success or failure of the 4th IR hinges on the quantity and accuracy of big data for each of these factors. In particular, it depends on how much accurate information each individual has about the ingredients contained in each food. The hardware and software elements do not require further research in the food science industry as long as the basic concepts are understood. The hardware and software industries continue to advance at breakneck speed thanks to electronic engineers and IT (information technology) experts, and Korea ranks among the global leaders in terms of hardware.

However, generating big data about food is a task that cannot be performed by those in the hardware and software industries. Because this represents data about diet and lifestyle, it can only be produced through fields such as agriculture, food science, bioscience, and life science. In this era, foodomics and life science technology are required to generate the necessary data about healthy living and lifestyles. More specifically, the technology required includes biotechnologies such as hormesis, whole genome sequence analysis, nutrigenomics, metabolomics, nutrigenetics, nutriepigenetics, and microbiome technologies, as well as foodomics disciplines such as sensomics, gastronomy, culturomics, dietary style, leisure style, and even "sikdanomics (sikdan is food table which is composed of main dish and side dishes)." Of course, there is no need to develop such core technologies within the food science sector. Data about the value of Korean foods should be generated by obtaining a clear understanding of these modern biotechnologies and applying them appropriately, while at the same time keeping up with the rapid pace of development and change in global biotechnology. There are also many aspects of food-related life science that require research before existing biotechnologies can be applied to our lives. Many countries are already hard at work utilizing these core technologies to generate big data. Although many believe that big data from other advanced countries can be easily imported to Korea, this is incorrect due to the food, cultural, ethnic, and dietary differences between Korea and other countries. 
From the perspective of diversity, the Korean food industry is well placed to play an important role in the development of personalized diets and could become a global leader if quality big data can be generated. Korea has a wide range of natural foods because of its long agricultural history, and Korean food has many advantages when it comes to responding to diverse demands for personalized diets.

This paper aims to deliver an accurate understanding of the 4th IR and point out errors and other issues with the Korean government's current 4th IR policy. As a key sector in the 4th IR, this study aims to approach personalized foods from the perspective of science and the social sciences, while also providing a brief outline of the biotechnologies and life science technologies required to produce such foods. Finally, this paper discusses some policy recommendations for the Korean government.

\section{The food industry and the Fourth Industrial Revolution \\ Characteristics of the food industry Low appropriability}

Most people are familiar with foods and the food industry. It is rare to come across someone who knows nothing about these subjects, and the majority of people can entertain a discussion about food and the food industry. However, this also means that there is a greater amount of misinformation about the food industry. Some people believe that the restaurant business and the food industry are among the easiest industries to understand. Although this is technically correct, it also causes a number of problems. In reality, the food industry is not as technology intensive as people thought, nor does it have a high level of technological protection or high barriers to entry [6]. This can be summarized as low appropriability [7]. Development in the food industry is not driven by production and technological advancement. In developing countries, the longstanding problem is food supply and agricultural production because of food shortages. In the industrial era, the food industry was able to grow simply by increasing production, and the industry was capital-intensive and production-focused. However, in the future, industrial production will be the problem, and the new focus will be on meeting the demands of consumers. Consumers are interested in many other factors outside of a full stomach, including taste, culture, health, and happiness, and a purely technological approach to food production cannot satisfy these demands. Accordingly, the government's current food industry policy, which is skewed towards development and production, will not be able to turn Korea into a global leader in this field. The government's focus on R\&D has led to a situation where profits are monopolized by only a few companies, and the first step towards a solution is addressing this problem. The goal of national food R\&D should be promoting health, happiness, and national prosperity instead of chasing corporate profits through technology transfers.

The Third Industrial Revolution was able to provide answers through production, but the commercialization, automation, and standardization of the production economy cannot satisfy a diverse range of consumer needs. The 4th IR will enable companies to satisfy consumers with different characters and preferences, and this is the only way forward for the Korean food industry.

\section{Public nature}

A nation's food industry is closely intertwined with the health, safety, and happiness of its people, as well as the national economy $[6,7]$. This is why both governments and the public are very sensitive about issues of food safety, and governments closely monitor the price of food and food products. Accordingly, unlike in other industries, it is rare for a company in the food industry to have monopolizing power, and even if such a company exists, it cannot abuse this power by raising prices as it pleases. Food prices have a substantial influence on inflation across the whole economy. This is why governments seek to control food prices in an attempt to exercise influence over inflation. It is also important to keep in mind that the safety and healthiness of foods are important criteria for judging public health and wellbeing in a country. A few years ago, a large Korean company released a new product developed with quality materials, but were asked by the government to lower the price after complaints that it was too expensive. This is one of many instances where companies released a new product but then abandoned it shortly due to government price control.

This means that unlike in other industries, it is difficult for companies in the food industry to make a fortune by developing and manufacturing a new product, but the government and scientists continue to focus the majority of their attention on product development as the main driver of growth in the food industry. When viewed in this light, the government's current food development and production policies are misguided and ill-fitted to meet the needs of society in the future. On the other hand, the government has no way of controlling personalized diets that have been designed to match the specific preferences and cultural and biological traits of individual consumers. This is why personalized diet is a high value-added industry.

\section{Endless choice}

The end consumers of the food industry are ordinary people. Although the same applies to many other industries, ordinary people are not the end consumers for 
pharmaceuticals. In this case, it is doctors that make up the end consumer group, while the end consumers for machines and equipment are companies. This is why ordinary consumers do not have a "right to know" when it comes to pharmaceuticals. However, because foods are consumed by everyday people, they have the right to know about the safety, health benefits, and origins of the foods they consume. Consumers also have the right to choose. The same applies to automobiles, smart phones, and home appliances, for which ordinary people are the end consumers. However, cars and cell phones are generally used for 5-10 and 2-5 years, respectively, so it takes a long time until consumers are presented with another purchasing decision. Once a consumer buys a car or phone, they will not have to make another decision for at least several years. On the other hand, consumers have to make at least 200-300 choices, or sometimes up to 1000 choices every year when it comes to foods. Because choices about foods are so frequent, consumers want to know a lot about foods and exercise their right to know. The rights to know and to choose are important factors that need to be taken into account in discussions about growth in the food industry.

Accordingly, the factors that consumers take into account when choosing foods should be part of the discourse as well. Korea has a history of focusing only on the supply side when it comes to discussing consumers' right to choose. In other words, the majority of attention was focused on nutrition to prevent people from going hungry, prices that consumers could afford to pay, and distributing food in a way that prevented them from getting sick. The government remains stuck in the paradigm that corporate enrichment equates to development in the food industry. However, in the future, industrial development that caters to corporate profits will not be sufficient to achieve true growth in this industry. Instead, it will be necessary to pay more attention to taste, gastronomy, and healthy lifestyles. It is also necessary to have an accurate understanding of the criteria that consumers consider when choosing between foods. There are three key differences between foods and pharmaceuticals when it comes to consumer choice.

\section{Unique characteristics of foods}

Taste Even though health has become an important criterion for making decisions about food, taste remains as a king criterion. In the industrial era, the importance of taste caused many companies to add sugar and salt to their products to make them more flavorful, but this led to a number of severe side effects including obesity. In scientific terms, salt and sugar are the tastiest substances on earth. When combined with greater calorie counts, people's desire to eat tasty food has led to an obesity epidemic and a host of obesity-related diseases including diabetes and cardiovascular disease. In the USA, the epicenter of the global food industry, the obesity rate has soared to above $60 \%$ and become a serious social problem. Taste rules when it comes to choosing foods, and future growth in the food industry will depend on how well companies are able to develop products that are full of healthy flavor without the side effects of causing obesity and metabolic diseases. This is why the food industry needs to adopt an approach that looks beyond technology and production.

Since the 1980s, the food industry in Korea has been treated as less important than other sectors such as pharmaceuticals, as it is considered less technical and less worthy of academic attention. Those speaking about the importance of biotechnology in the food industry have tended to be ignored by pharmaceutical scientists, and even by bureaucrats in terms of priority for understanding and granting. However, in this day and age, foods are one area that cannot be touched by experts in pharmaceuticals or biotechnology. This is because foods have certain characteristics that are not present in pharmaceuticals. It goes without saying that the first of these traits is taste.

Culture Culture is another unique aspect of food. During the industrial era, when technology-based food production was the main goal, culture was not taken into account. However, a closer examination of the food industry reveals that culture is a key factor in consumer choice and very important for development in the industry. The Korean food industry cannot develop into a global leader without cultural support. Despite this, the Ministry of Agriculture and Foods (MAF) currently has no plans to research this aspect of food due to the influence of some scholars who spread inaccurate information. This misinformation is hindering growth in the food industry. The MAF needs to move away from its current paradigm which caters to product development by a small number of companies. Without a paradigm shift, the Korean food industry will be unable to grow.

Taste fatigue During the 2000s, a number of pharmacy professors were appointed to work on research projects to fight obesity and extend healthy life expectancy, but their poor understanding of food led to a number of errors. The most glaring mistake was coercing companies to develop "obesity prevention" products, based on the false belief that food could be treated as a kind of pharmaceutical. They asked companies to develop products to "prevent" obesity or even "prevent" air pollution caused by yellow dust. These scientists believed that by eating such products, everyone would be able to live to 100. However, even if such a product could be developed 
and distributed to every person, those people would die much faster than their peers. This is because even if an "obesity prevention" product were very effective, anyone forced to eat it only would grow sick of the taste after a few days and prefer to starve to death than eat nothing but that product for the rest of their lives. Unlike with medicine, people grow tired of the taste of food over time. Even now, Korean bureaucrats continue to include "preventing something" as part of indexes for evaluating projects, despite the fact that this is based on a false understanding of foods. This is a foolish policy.

Developing foods that can satisfy the diverse cultural, biological, and lifestyle needs of consumers is the key to global success in the future, but bureaucrats seem hellbent on promoting the development of monotonous foods rooted in an outdated industrial era mindset. The Korean food industry will struggle to develop unless this changes. It is regrettable that this line of thinking still underpins many government projects. The government needs to rapidly abandon its corporate-oriented food policy and replace it with one that caters to the public.

Because consumers make hundreds of choices about foods every year, choice is the key for both lifestyle and life itself. It needs to be recognized that Korea's current approach to product development and production is undermining growth in the food industry.

\section{Life style science and Technology for the Public}

As discussed earlier, the food industry is an integral part of our lives. Accordingly, even if every other industry developed by humanity falls by the wayside, the food industry will remain as long as humans still exist on earth. Although ordinary people are the end consumers of smart-phones and cars, these industries are not considered part of life science. This is because once a person has decided between buying an Apple or Samsung phones, it has very little impact on their life thereafter. The same applies to choosing between different brands of cars. On the other hand, decisions about food need to be made every day, and the foods a person chooses to eat will influence their taste, health, life, and even happiness. This is why the food industry is one of the life style sciences [8], and why this industry should be approached from the perspective of life style science technology that can enrich people's lives and make them happier and healthier, instead of industrial technology with a focus on production. Such an approach focuses on foods that are tastier and healthier and help people to live happy and healthy lives. It also dictates a greater focus on the consumer economy, respecting consumers' right to know and ensuring that consumers have access to healthy, safe foods that improve their quality of life.

For example, when it comes to food safety, the status quo is biased towards developing technologies such as speed radars to catch speeding vehicles on expressways, and search vehicles that are found to be speeding on multilane roads. Meanwhile, companies that produce speed radars are only interested in increasing sales. Companies are ultimately obsessed with the bottom line and profit indicators. But money should not be an issue when it comes to public safety. Even if it costs extra, software systems should be built into hardware that allow ordinary people to detect and prevent safety threats, and public safety should be the primary focus of software development. Rather than continuing to develop new products or technology, a paradigm that combines existing technologies to contribute to public safety and quality of life is paramount.

The food industry also needs to shift its goals towards public health and cultural development. It is time for the government to step up and generate scientifically verified big data to improve public health and safety, as well as promote access to accurate information. There is no time to waste on product and technological development, and the development of technology that pools data together is not a job for food scientists anyway. The government should view generating big data and providing such data to individuals and companies as a top priority, as data is crucial for helping both groups make better decisions.

The ultimate goal of the state should be ensuring that people live happy and healthy lives instead of encouraging competition through endless work.

\section{Personalized diets in the fourth industrial Revolution era} An accurate understanding of fourth industrial Revolution

The term "Fourth Industrial Revolution $\left(4^{\text {th }}\right.$ Industrial Revolution)" was first used in "Industry 4.0," one of ten major projects included in the German government's "High-Tech Strategy" released in 2010. It refers to convergence between the manufacturing and IT industries. In 2016, "Mastering the 4th IR" was chosen as a main theme for the Davos Forum held in Geneva [5]. This was the first time the Davos Forum, which is known for covering economic issues such as low growth, inequality, and sustainability, had dealt with a topic related to science and technology. The 4th IR then became a talking point around the world when it was added to the World Economic Forum (WEF)'s agenda, and many futurologists and research institutes worldwide have since discussed the industrial and social changes that the 4th IR will bring about.

As an advocate of the 4th IR and WEF Executive Chairman, Klaus Schwab defined the revolution as "A technological revolution that builds on the Third Industrial Revolution and is characterized by convergence between digital technology, physics and the bio-industry, bringing about rapid changes to economic systems and 
social structures" in his book 'The 4th IR.' He wrote "We are on the cusp of a technological revolution that will fundamentally change the way we have lived and worked until now. The scope and complexity of this change will be unlike anything humanity has ever seen before." The First Industrial Revolution (1760-1840) was marked by production through machines due to the invention of railroads and the steam engine, while the Second Industrial Revolution (late nineteenth century-early twentieth century) enabled mass production systems through the invention of the electric light and production lines. The Third Industrial Revolution led to the advent of the information era through semiconductors and mainframe computers (in the 1960s), personal computers (in the 1970s to 1980s), and the Internet (in the 1990s). Building on the first three revolutions, the 4th IR will be one of super-connectivity and hyper-intelligence. It is expected to bring about a more advanced society by connecting people and objects together through ICT technologies including the IoT and cloud computing, as well as big data and AI.

This has also caused controversy about the validity of the generational concept put forward by Schwab. Rifkin [3], who was the first to use the term Third Industrial Revolution, has stated that it is too early to talk about a "Fourth Industrial Revolution." He believes that the amazing changes currently taking place are merely an extension of the information revolution brought about by the Third Industrial Revolution.

In this sense, the 4th IR has been mainly discussed in terms of social and economic changes in the postindustrial era, while in Korea the discussion has been limited to topics such as production, efficiency, and convenience. This is because the Korean government believes that economic growth in Korea hinges on developing the industrial economy. However, this misses the point of the 4th IR. Although there is a lot of talk about the advent of autonomous vehicles due to advancement in AI, the goal of developing autonomous vehicles in the US is to improve the quality of life for elderly or disabled people who cannot drive, while in Korea the focus remains squarely on how to produce and sell more autonomous vehicles. This is based on the misconception that all cars will be autonomously driven in the future, an argument which ignores the fact that there is both life style and pleasure in driving and treats humans as slaves to automation. The 4th IR is about more than just efficiency and producing autonomous vehicles that are able to beat the competition. It is about the changes to lifestyles and social structures that will be brought about by technology such as autonomous vehicles. In the same vein, the discourse about the 4th IR in the agricultural sector is mainly focused on production and distribution systems such as 3D printing, smart farms, and smart logistics. But this focus on production is merely an extension of the Third Industrial Revolution rather than a true 4th IR. In a future with advanced AI, humanity also runs the risk of losing the very things that make us human. This is why the 4th IR needs to be driven in a direction that improves quality of life and human happiness.

Elements of the Fourth Industrial Revolution The 4th IR can be understood as a revolution in industry and lifestyles that is fuelled by AI and life science. Humanity needs to ensure that advancement in $\mathrm{AI}$ is accompanied by greater human happiness instead of allowing machines to achieve dominance by taking away human intelligence. There is a lot of confusion between AI and the 4th IR among the general public. It must be remembered that AI and life science refer to technology while the 4th IR refers to lifestyle. Accordingly, an understanding of AI is required to make sense of the 4th IR. Rather than replicating human brains, AI should be viewed as learning how humans think and the processes that make thought possible [9].

\section{(a) Hardware factors}

AI needs to be equipped with hardware similar to the neurons and genome that enables humans to think and solve problems. There is no need for those working in the food industry to understand this hardware. However, the view held by certain bureaucrats that food science is not a core part of the 4th IR because those in the industry do not understand the technical aspects is misguided. These bureaucrats are unable to see past the outdated logic of the industrial era, believing that producing high quality machinery at a competitive price equates to economic development. Producing such machinery should be left to the digital and electronics industries, while the food industry need only apply this technology to create improvements in quality of life in terms of happiness.

\section{(b) Software factors}

The 2012 Davos Forum in Geneva, hosted by the WEF, was held under the theme of the importance of soft power. The power of AI software lies in building on the mechanical aspects by enabling machines to recognize their surroundings, environment, and behavior so that they can put this data together, draw conclusions, and take action, in other words, creating systems that allow machines to act like humans. In this sense, AI is a discipline that cannot develop without an accurate understanding of the biological processes that underpin the mechanisms of the human brain. The core AI technologies involve developing super-connected and hyper- 
intelligent algorithms and programming AI systems. Similar to hardware, those working in the food science industry need only understand the basic principles and do not require detailed knowledge of the technology. A lack of knowledge about these systems does not mean that the food industry will be left behind in the 4th IR. Instead, the task for the food industry is applying these systems to successfully produce personalized diets and help develop Korea's food industry into a global leader.

Putting it this way may give the impression that the food industry can sit back and do very little in the 4th IR. However, this view is based on a lack of knowledge about the characteristics of the food industry. The Presidential Advisory Council on Science on Technology in Korean Government decided to exclude the food industry from a list of ten core 4th IR industries on the grounds of being unrelated, but this decision is rooted in an incomplete understanding of the importance of big data in the food industry. These bureaucrats know nothing outside of hardware, software, and the production of AI machines. Personalized diets will be a core industry in the 4th IR, so it is important that the government has a clear understanding of what the revolution is all about. This paper discusses why personalized diets will play an important role in the 4th IR.

\section{(c) Big data}

Even with top quality machinery and software, AI cannot function without data. Because the Third Industrial Revolution was based around centralized control with a single correct answer to each problem, the only thing required was the data and equation to perform the necessary calculations. In this context, an abundance of information can actually hinder calculations and make things more difficult to control. On the other hand, the advent of more powerful hardware in the 4th IR means that a lack of information is now fatal to making accurate decisions. A large number of data points are required for optimal decision-making. Accordingly, accurate and error-free data sets are an essential part of AI. Cuttingedge hardware and software are largely meaningless unless they are supported by a sufficient quantity of data. Success or failure in the 4th IR is dependent on big data. Although the term big data is used, the accuracy of the data is often more important than its quantity. This is why it is important for governments to filter out noise and fake data.

Unfortunately, despite the Korean government's fanfare about the 4th IR, no investment is currently being made in big data generation or filtering out fake data. The Ministry of Agriculture and Foods spends in Korea Government less than $1 \%$ of its R\&D budget on big data generation, while this figure should be above $30 \%$ in order to properly prepare for the future. In discussions about data generation, there are inevitably some bureaucrats who argue that using data from other advanced nations such as the USA will suffice. However, even if the hardware and software systems of other countries can be imported, data about foods, food products and the personalized diets most suited to individuals cannot be used due to the differences between Korea and other countries. In fields such as life science, data obtained from Americans will not work in Korea. Korea not only has different foods, but other differences exist in lifestyles, biology, and traditional foods. In order to develop Korean foods into global products, data about such foods must first be obtained. This means data about Korean culture and the Korean people. Without generating big data applicable to Korea, the country has no chance of succeeding in the 4th IR. This is an example of soft power.

\section{Characteristics of the Fourth Industrial Revolution from the perspective of the food industry}

(a) Decentralization

The 4th IR involves a transition from production systems and integrated information systems designed for efficient production towards hyper-connected systems that can produce a range of personalized products catered to individual consumers. Accordingly, this is not an efficiency war aimed at competing on price or production. Decentralized systems are a leading example of an economic model designed for consumers. For example, a block-chain system that generates clean energy for a specified consumer block in a certain region and sells any leftover energy. This kind of block-chain system can categorize consumers into blocks based on their biological traits, preferences, culture, or history to deliver products or systems that meet their specific needs. Of course, further development in AI and big data is needed to reach this level of decentralization, but technological advancement could eventually move beyond block-chain to create P2P (person to person, product to person) models with greater levels of stratification and diversity. In this sense, decentralization is a core element of the 4th IR.

\section{(b) Personalization}

If sufficient quantities of data are generated about each individual and object, deep learning technology will be able to put the pieces together to enable the provision of personalized hobbies, culture, travel, relaxation, healthcare services, and foods for individuals instead of groups or blocks of consumers. Advancement in life science could even enable distinctions to be drawn not only 
between individuals, but also between the emotions and biological rhythms within an individual. It would also make personalized services possible that help each person find their own happiness, the ultimate pursuit of all humans. Of course, this is conditional on the generation of suitable data, but if this can be achieved then the food industry would be able to provide personalized P2P diets or foods based on an individual's lifestyle data and even genetic data. In this way, personalized diets that help individuals stay healthy will be a key industry in the 4th IR.

Those who believe the 4th IR is all about production through automated technologies such as 3D printing and smart farms suffer from the misguided view that it is a production revolution, when in reality it is a revolution in personalization. Instead of manufacturing imitation foods with 3D printers, using the technology to produce customized human organs that function as nerves, veins, or muscles based on big data analysis is a true application of the 4th IR. It must be remembered that as the 4th IR unfolds, shifting towards natural and sustainable foods is the path towards human happiness. Similarly, instead of focusing on technological factors such as facilities, the production environment, and automation, discussions about smart farms in the 4th IR should center around the use of block-chain technology to supply consumers with a diverse range of specialized products through processes such as "hormesis" that can fulfill their biological and cultural needs.

\section{(c) Value sharing and sharing economy}

In the 4th IR, all data and things, especially values and facilities, could be connected and shared specifically person to persons, and person to things due the development of super connectivity by AI and IoT. Thus, sharing economy will be fully activated and creation of shared values will be more important for new sharing economy.

\section{(d) The first lifestyle Revolution}

In Korea, lifestyle revolutions have come about from the introduction of technology that enabled the country to join the ranks of advanced nations in terms of mindset and sophistication. For example, the introduction of showers in every home led to better disease prevention and improved quality of life. In the same way, the 4th IR will bring about lifestyle changes that ultimately lead to greater human happiness. This is why the 4th IR can be viewed as the first lifestyle revolution. Until now, the Korean economy has been measured through production indexes such as GDP and GNP. However, dramatic growth is no longer possible in these terms. In order to become a truly advanced nation, Korea needs to take the lead in developing new lifestyle indexes that measure public happiness and prosperity.

The Korean food industry has a solid foundation compared to that of other countries and is therefore well placed to be competitive in the global food industry. It is unlikely that the USA will be a leader in the food industry of the future, as the country does not have a long agricultural history and produces food which is lacking in both tastes and culture.

The 4th IR will be the first lifestyle revolution. Because the food industry is closely connected to lifestyles and consumption, it will serve as the cornerstone of the 4th IR in the future. Human thought and behavior is influenced by emotions and cannot be explained entirely through rational decision-making. Accordingly, AI and other 4th IR technologies that drive big data and deep learning will facilitate the introduction of personalized and decentralized services. This means that the main focus will be designing unique lifestyles instead of production. Accordingly, it is regrettable that discussions about the 4th IR in the agricultural sector pay little attention to values and how the food industry can develop in a way that promotes happy and healthy lifestyles and social structures.

\section{The Fourth Industrial Revolution and personalized diets Personalized diets based on individual genetics}

All humans wish to live healthy and full lives. It is human nature for people to begin paying more attention to health and take steps to manage their health as they get older. This holds true across all ages and all cultures. Korea has already become an aging society. In the future, 4th IR technology will be applied to the food industry. For example, when providing personalized diets, companies may be able to obtain genetic information about people through deep learning to make accurate predictions, then provide dietary guidelines to individuals based on their nonen style (leisure style) and dietary style to maximize their health and happiness.

As biotechnology continues to develop, we will be able to understand not only the entire human genome, but also how individual genes are connected to the expression of certain diseases. Many genetic factors are related to health and disease, and we now know that they play a dominant role in determining certain aspects of human health and longevity. In the future, genetic analysis will reveal which individuals are susceptible to certain diseases. Because the primary structure of DNA does not change in response to lifestyle or the external environment, structured big data about individual genomes will build up over time. If this kind of structured data is generated alongside big data about the foods of each country, it will be possible to produce a range of personalized diets. The problem is that although advancement in 
biotechnology has led to large quantities of data about genetics, health, and susceptibility to diseases, more data is required about the chemical, biological, and agronomical features of Korean foods and cooking methods specifically. The lack of such data is concerning as it could lead to poor decision-making that causes Korea to miss out on an opportunity to play a larger role in the global food market. Nevertheless, although big data of this nature is currently unavailable, data about the genes of individuals can still be used to provide some degree of personalization for medicines and nutrition.

\section{Personalized diets based on lifestyle habits}

What do people do to stay healthy? Ironically, most people eat and do whatever they please while hoping to remain in good health, forgetting the fact that it takes a lot of patience and effort to stay healthy [10]. More specifically, people wish to live long and healthy lives but fail to take steps to make this happen. Lifestyle is crucial when it comes to remaining in good health. Lifestyle can be broadly divided into three categories: working style, nonen style (leisure style is one of nonen style), and dietary style. It is possible to add "sleeping style" to this list, but because lengthy scientific explanations are required to understand the way in which people sleep, this factor was omitted for the sake of brevity. Since many people do not have much control over their working style, and there are fewer differences between individuals, this has less of an impact on health. On the other hand, nonenstyle (leisure style) and dietary style have a much greater influence on health and longevity.

The Korean verb non-da can be translated as "to play" in English, but this is not a complete translation. Words such as exercise, enjoy, be idle, be lazy, healing, rest, and gamble also fail to capture the meaning. The verb nonda implies relaxing both mentally and physically in a way that heals the body. It also means to enjoy oneself and have a good time. On the other hand, non-da can also have a more negative connotation that denotes wasting one's time without doing anything productive. The Korean people used to have an appreciation for the arts, but at some point the culture began to place less value on having fun, exercising, and resting, and instead focused solely on working. This is largely due to the industrial economy. In any case, the verb non-da is used to refer to time spent on activities other than eating, working, and sleeping. Of course, leisure is generally not something that is formally taught. Knowing how to relax is important because our leisure style is closely connected to our health and lifespan. Non-da is a word that illustrates part of the Korean worldview. In this sense, "noneun style" does not simply refer to the way a person "plays" or engages in leisure. Since leisure style, time spending style, has a large impact on our health and lifespan, learning how to make proper use of our leisure time is also very important. Of course, learning to use your leisure time in a healthy way is a difficult process that requires patience.

Third, it is necessary to improve our dietary style to live a long and healthy life. This refers to the type of foods we eat and how we eat them. The food that we choose to eat on any given single occasion is less important because dietary style includes both cultural and historical factors. However, most people only focus on what they eat and pay little attention to improving their lifestyle, which requires more patience and effort. They are reluctant to change their noneun-habits or eating habits, hoping to become healthy through food choice alone. Of course, it is true that the substances contained in the food we eat matter, there are correlations between nonen style and dietary style, and the noneun activities that accompany eating have cultural importance.

Since many people do not have the chance to determine their own working habits, and noneun style is not something we formally learn, these habits can be difficult to change even if we invest money into doing so. However, eating is necessary for survival. Whether rich or poor, most people eat three meals a day. This means that everyone has three opportunities per day to fix their dietary style. Eating habits can be changed through conscious effort. In order to help consumers improve their dietary style, they need to be provided with accurate information about foods, including safety, functions, traditions, and culture. There is currently a lack of information in this respect. Because this kind of information is in the public interest, governments should invest in research to create it if no such data exists. Without accurate, useful, and up-to-date data, it is impossible to provide services that help people improve their diet. Most experts believe that improved eating habits and lifestyle, as well as healthy food choices, can extend an individual's lifespan by 5-6 years. Furthermore, if children are taught about healthy noneun habits when they are young, it can add another few years to their lives. As an aged society comes, the number of people involuntarily engaging in noneun time (people not-engaged in works) increases.

This means that depending on our lifestyle, the foods we eat and the way in which we eat those foods is very important. In ancient times, people ate different foods for breakfast depending on what they had for dinner the previous night. In Korea, people would eat haejangkuk with bean sprouts rich in aspartic acid if they had been drinking the night before, or haejangkuk with vitaminrich ox blood if they had stayed up all night working. In this sense, Koreans have been eating "personalized fit" food products for thousands of years. If advancement in biotechnology and AI can be linked to food and 
individuals, it will be possible to deliver personalized diets that cater to each individual's lifestyle and preferences, allowing everyone to live to 100 in good health. Although leisure and dietary style are individual habits, they contribute to public health and longer life-spans, which reduces medical costs and lowers the healthcare burden on the government, ultimately contributing to greater fiscal soundness. We have to learn how to lean about good noneun habits and best dietary style. This is why noneun leisure and dietary style are crucial to living long and healthy lives. They are closely linked to personalized diets.

\section{Epigenetics and personalized diets}

Even as adults, people tend to like the foods they ate as children and even the foods that their mother enjoyed while carrying them. In Asian countries, it is believed that eating habits are passed down between generations and that a person's favorite foods are determined based on the traits of their mother. Many people view this as unscientific, pointing out that eating habits cannot be genetic as genes are not affected by diet. However, modern biotechnology has shown that many lifestyle habits, including eating habits, are hereditary. Although lifestyle habits do not affect the primary structure of DNA, they can impact the proteins that combine with DNA (histones) or cause methyl groups in $\mathrm{OH}$ radicals in DNA side chains to be removed or added. In this sense, eating habits are related to genetics, and this discipline is called epigenetics.

It has also been discovered that diet has a large impact on the distribution of gut bacteria, which in turn affects gut health, immunity, overall health, physical constitution, and even "guts" in the colloquial sense. This biological phenomenon is called the microbiome. Accordingly, collating big data on how certain foods or ingredients from different countries affect the distribution of gut bacteria is an important part of designing and providing personalized diets. Although food cannot change our genetic information, it can have an impact on the microbiome and epigenetics, and this influence can carry over between generations.

In a similar vein, although data is limited at present, there is some evidence that noneun style and dietary style have an epigenetic impact and cause changes in the microbiome. These epigenetic habits can also be passed on to future generations. This kind of unstructured data is an integral part of big data as it can be used to design personalized diets that vary based on the each individual's lifestyle and the environment they grew up in.

In scientific terms, bacteria distribution and epigenetic changes are related to sense of taste, which in turn is linked to eating habits and ultimately overall health and wellbeing. These factors also impact other biological elements including our genome, proteome, and RNA and are connected to health and even general feelings about life. In other words, the microbiome and epigenetic changes are linked to many other phenomena including chromosomes, mitochondria, DNA, genes and genomes, the brain, and our habits. This is similar to the deep learning mechanism in AI and can open the era of real personalized diet in future.

In addition to epigenetic and microbiome data, big data on food diversity will be equally important. Alongside accurate data about the genomes and environment of each individual, generating a variety of accurate and up-to-date data on the taste, historical, geographical, agronomical, and cultural characteristics of foods is a necessary precondition for the 4th IR. Unfortunately, at present, the Korean government has shown little interest in generating big data about foods, while the agricultural industry remains focused on R\&D and production. This is a misguided policy that fails to consider the future and is stuck in an outdated paradigm of industrialism. This pattern of favoring short-term benefits over longterm paradigm changes is common among government bureaucrats. As the Korean government catches on to the importance of the 4th IR, they have launched a number of convergent research projects. However, the government believes that establishing platforms by combining foods and biotechnology research with ICT is sufficient to achieve success, when in reality the Fourth Industrial Reality requires far more than just platforms. These efforts will prove fruitless unless combined with data about lifestyles, including biological data. The government needs to promptly change its stance on this matter.

\section{Personalized diets and life science} Why is modern biotechnology important?

How and where can the big data described above be generated? Producing personalized diets to meet the demands of the 4th IR, requires many technologies (superconnectivity, AI, the IoT), but these will not be developed by the food science industry. The same is true of biotechnology. All that is required is applying this cutting-edge technology to produce personalized diets. We live in an age of science. Personalized food promises to be an industry that can help people live happy and healthy lives through life and diet. The data of life and diet are closely related on life science of human, and this big data can be generated through modern biotechnology. In other words, the personalized food industry is at the forefront of modern biotechnology. Fake data is of no use to the personalized food industry and can actually serve as an obstacle to discovering the truth, but biotechnology also has the capacity to filter out such fake data. 


\section{Understanding modern biotechnology}

The author has coined the term foodomics to refer to biotechnology related to foods. This term encompasses a wide range of biotechnologies including whole genome sequence analysis, nutrigenomics, metabolomics, nutrigenetics, nutriepigenetics, and microbiome technologies, as well as gastronomy, sensomics, culturomics, dietary style, noneun style, and even "sikdanomics," which embodies the Korean diet. Another form of technology required for producing personalized agricultural products is hormesis. As mentioned earlier, although the food industry is not responsible for developing such technologies, a basic understanding is required in order to apply them to generate big data.

\section{Hormesis: technology for producing personalized farm produce}

Plant bodies, the substances that make up foods, exhibit a hormetic reaction upon receiving microstimulation or nutrient stress. This phenomenon can be used to boost antibiosis within the plant body or promote the proliferation of substances that serve a health function. This technology is called hormesis [4]. When the power of hormesis is effectively harnessed, DNA stimulates the organism to a restorable level, manipulating metabolism within cells and thereby inducing the plant body to remain in homeostasis. This can be utilized for industrial purposes such as increasing the production of phytochemicals.

\section{Requisite modern biotechnologies for personalized diets Whole genome sequence analysis}

The development of NGS (next-generation sequencing) technology and other biotechnologies has enabled a person's entire genome to be analyzed at a low cost, and also shed light on how specific genes (SNP) are connected to certain diseases. The analysis of genetic makeup has facilitated the development of personalized medicine and nutrition by grouping people according to body type or susceptibility to certain diseases.

\section{Nutrigenetics}

Although it is well known that eating food provides calories through the nutrients contained inside, modern biotechnology has revealed that the impact of food on DNA expression has a greater influence on health and biological phenomena within the body than the nutrients themselves. Accordingly, learning about the expression of different genes allows us to determine the health effects of eating different foods and ultimately develop personalized diets as big data accumulates in this field.

\section{Proteomics}

As described above, the food we eat influences DNA expression. In turn, this impacts the tRNA signal transduction system, causing several proteomes to be synthesized. These proteomes regulate growth and balance within the body. Accordingly, they can be used as a mechanism for bodily regulation through food choice and also to generate more data about food and health.

\section{Nutrigenomics}

According to nutrigenetics, although food has an impact on genetic expression, the differences between the genetic structure of individuals (SNP analysis, gene sequencing) also cause bodily changes in metabolomic expression when food is consumed. This means that nutrigenomic data obtained after consuming different foods or nutrients is an important part of creating personalized diets.

\section{Metabolomics}

The fact that food has a nutrigenomic and proteomic impact also influences bodily activity and metabolism. Accordingly, researching the metabolomes found in urine or blood can give insight into the bodily changes that occur during daily life. Lifestyle also has an impact on metabolomic expression based on the differences in genetic structures between individuals. Obtaining more data about metabolomics could facilitate a greater understanding of how lifestyle impacts health and open the path towards disease prevention in the future. This is why metabolomic big data is a key to developing personalized diets.

\section{Nutriepigenetics}

Although lifestyle habits do not affect the primary structure of DNA, they can impact the proteins that combine with DNA (histones) or cause methyl groups in $\mathrm{OH}$ radicals in DNA side chains to be removed or added. This discipline is called epigenetics. It is now known that many lifestyle habits, including eating habits, have an epigenetic effect that is passed between generations. The unstructured nutriepigenomic data obtained from these changes is also of great use in developing personalized diets.

\section{Microbiome}

Studies have shown that the food we eat not only impacts our genes, but also the genomes of the bacteria that live in the gut, ultimately altering the distribution of gut bacteria. This biological phenomenon is called the microbiome. The gut microbiome is related to health and immunity. Although food cannot change the genetic make-up of gut bacteria, it can cause changes in the 
microbiome. This is why big data about the microbiome is crucial for designing and producing personalized diets.

\section{Bioinformatics}

Bioinformatics is a core technology built on the foundation of big data obtained through modern biotechnologies. It is used for predicting an individual's biorhythm, designing biological systems, and developing alternative systems by biological big data, and these can be controlled and optimized through personalized diets and changing life style. This is an important aspect of software for algorithm development.

\section{Life science technology: convergent foodomics for personalized diets \\ Sensomics}

Although food has many characteristics including flavor, fragrance, and texture, the most notable feature is taste. Modern science has added a number of additional elements such as nutrition and health functions, but the number one attribute of food is still taste. Taste can be defined as the sense that stimulates the palate and nose when food is eaten, as well as the receptors in the mouth that measure pain, feeling, and temperature. Sensomics refers to technology that treats taste as a living thing to be studied, thereby generating big data. This involves a number of scientific, sensory, chemical, and physiological disciplines and is highly sensory in nature as taste can vary between individuals. Taste is undoubtedly an important factor in personalized food because it is the main criteria by which the quality and desirability of a food is judged.

\section{Gastronomy}

Taste is king when it comes to choosing foods. Gastronomy refers to the study and pursuit of taste and is now joined by the field of molecular gastronomy due to advancement in food science and biotechnology. Gastronomy is concerned with the chemical changes that occur in the texture, structure, taste, and ingredients of foods as they are cooked and also studies the relationship between taste and function through molecular biology to produce new flavors and textures. Furthermore, shedding light on the relationship between taste, ingredients, and cooking is a fundamental part of food branding. Molecular gastronomy is already developing into an important field in consumer economics and life science in France, Italy, and Spain [11].

\section{Culturomics}

As described in earlier sections, culture is an important characteristic of food. Culturomics is the study of the traditional cultural elements embodied in food. In addition to history, the traditional knowledge and traditional techniques found in ethnic foods are important parts of culturomics. In some cases, seemingly irrational cultural practices play an important role in food choice. Traditional knowledge and culture are often intertwined with the development of ethnic foods in a certain region, along with traditional manufacturing techniques. Due to heightened interest in the health functions of foods, knowledge written down in ancient documents is being reinterpreted through the lens of modern biotechnology, generating big data through scientific verification. In terms of modern life styles and noneun culture, culturomics is an important discipline for promoting growth in the tourism industry and developing personalized diets based on local geographic conditions. Consumers want personalized diets that are healthy and also embody a variety of traditional techniques.

\section{Taste fatigue and sikdanomics}

One feature of foods that does not apply to medicine is that people get sick of eating the same thing. This is why personalized food must encompass an entire diet (bapsangomics) or software content (sikdanomics) instead of consisting of a single product. The system of food choice available in Korea have guaranteed consumers' right to choose. Unlike western cultures where people often eat a single food, Korea's traditional diet consists of bap (boiled rice) eaten alongside a variety of banchan (side dishes). Another feature of sikdanomics is looking out for one another, since numerous banchans are shared between different people at the table (bapsang). Accordingly, sikdanomics is about eating a variety of foods to avoid taste fatigue, maintaining a balanced diet, and providing many options for consumers to choose from. As more big data is generated, sikdanomics will become the main choice for a system of personalized food that guarantees consumers the right to choose between a variety of products based on their tastes, preferences, and habits.

\section{The outlook for personalized diets in the Fourth Industrial Revolution}

\section{The outlook for personalized diets in Korea}

Although Korea is a leader in hardware, the country is lagging behind in software, which is one of the key components of the 4th IR. Thanks to several decades of continued investment, Korea is now on par with global leaders in biotechnology, but still lacks software capable of drawing links between human health, foods, and data obtained through biotechnology. The Korean government is supporting many convergence projects, but most of these projects are focused on hardware convergence through technology and products, with very little research on integration between hardware and software, 
such as convergence between technology and lifestyle or technology and consumers.

Creating software to generate value and data is a key element of the 4th IR, but the government has so far been miserly in supporting such initiatives or other big data projects. This is because during the industrial era, Korea adopted a catch-up R\&D strategy to achieve economic growth in which technological development was more important than content or data, as the latter could simply be imported from other countries. Although the value of technical patents as intellectual property is recognized, intellectual property rights pertaining to data or content have not been treated with the same importance or respect and are still routinely ignored. However, it was predicted at the Davos Forum in 2012 that in the future, software will have a much greater influence on a nation's competitiveness than hardware. The Korean government needs to rapidly shift its paradigm. Without sufficient quantities of data about Korea, in particular Korean foods, ingredients, agricultural, and marine products, the country has no chance of succeeding in the 4th IR.

Fortunately, the Korean food industry possesses vast troves of resources that rival the capacity of any nation and have the potential to generate a lot of big data. Because Korea has a long agricultural history, the country is home to a variety of natural foods and numerous methods for preparing and eating such foods [6, 10]. Thanks to this agricultural legacy, Korean food is rich in flavor, culture, and history. Even under the lens of modern science, Korean food holds its own against traditional foods from any nation in terms of healthiness [8]. If scientific big data about Korean food were produced, the country would be well-placed to develop healthy personalized diets, and Korean foods have a number of advantages for satisfying the diverse needs of consumers. Korea has great potential to become a global leader in the personalized food industry, but to achieve this, food scientists and medical professionals need to work together to convince the government to lead the industry in the right direction.

\section{Policy suggestions to promote personalized diets in the Fourth Industrial Revolution}

In order to make personalized diets a reality, preparation needs to start now. Korea needs to give up on the idea of borrowing products or systems from other countries. Instead, the government should generate its own big data to facilitate the integration of personalized diets with the biological and sensory needs of Koreans, and develop platforms and services that can provide many types of personalized diets to consumers. The government is currently fixated on developing platforms and systems for production, but many of these developed platforms and systems sit idle without being used. This is like building a network of super-highways before there are any cars to use them. This is due to misguided evaluation standards which view the development of a product or platform as success in and of itself. In reality, products need to sell and platforms need to be used in order to be considered a success. The number of products that have been developed but failed to sell is rapidly growing across Korean companies. The government needs to abandon the complacent belief that simply building platforms is sufficient. Once sufficient big data is generated, companies will have no trouble creating and using platforms to earn profits. All the government needs to do is provide a basic platform model.

\section{Generating big data to promote personalized diets}

As discussed earlier, the key to success in the 4th IR lies in generating sufficient quantities of unique, upto-date big data about Korea. Some may argue that the private sector is perfectly capable of generating such data, but this is not a job for the private sector. Developing new drugs is a high-risk, high-return strategy, so it is commonly believed that government investment is required to manage the risk. This may be true, but the same does not apply to food. The private sector is unwilling to take on this task because the food industry is characterized by low technological barriers, low barriers to entry, low appropriability, a public nature, and the fact that consumers have to make frequent choices about foods. It is not that the private sector is incapable of generating such data, but if one company did so then they would be simply helping their competitors, because these public data should be opened immediately to promotion. This is why the private sector cannot produce publicly available big data. Looking after public safety and health is the government's job.

\section{The role of government}

Generating big data is the first task for the government, alongside serving as a "control tower" for communication between groups from various sectors including science, culture, consumers, and suppliers. However, in order to achieve this goal, the government first needs to abandon its industrial era logic and establish a clear direction. When generating big data, the government should continue to improve and filter the data so that consumers and companies have ubiquitous access to high-quality data. It is also the government's job to tackle legal and institutional problems and put 
appropriate mechanisms in place to help the personalized food industry develop. In addition to this, the government must contribute to improving quality of life by reviewing and monitoring the impact of personalized diets on public health and safety.

\section{The role of private companies}

The task of creating personalized diets by integrating big data with available platforms ultimately lies with private companies. Private company should collect the individual personal biological data in terms of structured and unstructured data based on modern biotechnology. These personal biological data cannot be open due to Personal Information Law or Human Bioethics Law. As these big data highly are conserved by law, private company collecting and creating these big data can get power in the personalized diet market.

Once the personalized food industry is established in Korea, the private sector also needs to work hard to become global leaders in delivering personalized diets that cater to different countries, ethnic groups, and traditions around the world. This industry alone has the potential to create large corporations.

Since Korea has great potential in the personalized food industry, if the industry becomes firmly established then it would encourage many companies that moved their production operations offshore during the industrial era to return to Korea. This would be a new form of reshoring.

\section{The role of consumers and new jobs}

Based on publicly available big data, ordinary consumers will make an effort to maintain a healthy lifestyle through personalized diets. However, due to the complex interactions between AI, biotechnology, the nature of the food industry and consumer health, "personalized diet designers," or "personalized diet planner" may be required to make sense of personalized diets. The topic of disappearing jobs often comes up in discussions about the 4th IR, but this is one new job that will be created [12]. In simple terms, "personalized diet designers" would function as insurance firms or insurance planners. These individuals would need to be trained to seamlessly integrate information from a variety of fields including biotechnology, AI, and super-connectivity.

In the future, the government will be called upon to put in place systems to train these high-level professionals. This will require specialized education and specialized training institutes. At present, universities do not have the capacity to provide this kind of education as they remain focused on product development. Unless universities are able to evolve beyond industrial era logic, there is a risk that they might distort or ruin such education if it is left in their hands.

\section{Legal systems for personalized diets} Obstacles and solutions

The 4th IR represents a convergence between cuttingedge technology, traditional knowledge, and biotechnology. Although the arrival of the revolution is inevitable, there are many obstacles to overcome along the way. Privacy concerns are one issue that will need to be addressed when generating data about individuals through biotechnology and designing personalized diets that cater to individuals. In fact, finding ways to protect personal privacy is of paramount importance. In addition to this, the revolutionary development and changes brought about in industry and lifestyles are guaranteed to produce stragglers who are unable to adapt to the changes. This will mostly be an economic issue, but it also relates to knowledge and social systems. As mentioned in earlier sections, unlike medicine, the personalized food industry will be part of the private sector rather than the public sector. This could lead to a situation where the rich are able to stay healthy by eating personalized diets while the poor experience deteriorating health as they are unable to afford these luxuries. This would widen the gap between the rich and poor in terms of not only health, but also happiness. In addition to this, societies may become more individualistic. Finding solutions to these issues will require a group effort.

\section{Requisite systems}

The food industry as it currently stands will require a legal framework in order to develop into a personalized food industry. Legal considerations will also need to be taken into account to solve the social and economic problems described above. Scientific and legal associations will be called upon to come up with proactive measures for addressing these issues. In addition to this, institutional mechanisms will need to be put in place to train personalized diet designers.

\section{Personalized diets and ethnic foods}

In the future, all countries will endeavor to move beyond the paradigm of industrialism. The world will leave the industrial era behind and enter a new age of AI. Instead of remaining fixated on mechanical efficiency, humanity will transition into a lifestyle economy centered around human happiness. Advancement in AI will lead to the advent of the 4th IR, which will revolutionize the way we live.

An accurate understanding of the food industry is required in order to discuss what the 4th IR really means for the industry. The food industry is not dominated by monopolies, and due to the industry's public nature, success cannot be achieved by trying to develop "blockbuster" products. The food industry is also part of life science and the lifestyle economy, since consumers make 
so many choices about foods. It is difficult to look beyond the logic of the industrial era without a clear understanding of the nature of the food industry.

The 4th IR is about decentralization and personalization. It is the first true lifestyle revolution. For the food industry, the ultimate pursuit in the 4th IR is public health, safety, and wellbeing instead of efficiency and competition. A nation's success or failure in this era hinges on its ability to generate big data that is accurate and error-free. It is up to food experts and the government to correct misinformation in the food industry. Big data generated through biotechnology needs to be scientifically valid to be useful as a core element of the 4th IR. Considering the public value of this data, it must be generated by the government so that the private sector can utilize it to create profits. At the same time, knowledge must be provided to consumers so that they can exercise their right to know and right to choose, ultimately improving the wellbeing of society as a whole.

For personalized diets, some obstacles need to be overcome with many potential benefits. Life ethics concerns must be addressed when generating big data through biotechnology, while mechanisms to protect privacy must be established when designing personalized diets that cater to the unique traits of individuals. In addition to this, other issues that require solutions are looking after those that are left behind in the wake of the industrial and lifestyle revolution, and the private nature of personalized foods. In other words, only the rich may be able to afford such products. This is a challenge that needs to be addressed.

The advent of personalized diets will soon be upon us. In the future, it appears that the government will require large numbers of professionals such as personalized diet designers. The government should start taking action now to prepare for this scenario. Universities and other educational institutions need to begin designing programs and training staff to teach courses in personalized diets. Experts in this field will need to be able to integrate information from a variety of disciplines including biotechnology, AI, and super-connectivity.

In coming post-industrial era, personalized diet should be a major food industry for human health and happiness. For the personalized diet, the competitive powers are from healthy, tasty, and historic diverseness. The big data will be come from ethnic diversity, historical culture, and geographical features. So each ethnic foods will be an important fact and will studied nation-widely. Diverse food materials and diverse cooking are very important criteria for personalized foods.

Korea is well positioned to become a leader in the market for personalized diets that help people live longer, healthier lives $[13,14]$. Because Korea has a long agricultural history, the country is home to a variety of natural ethnic foods and many methods for preparing and eating such foods. This means that Korean ethnic foods, in particular traditional Korean foods, are suited to meeting the demand for diversity in personalized diets $[15,16]$. Korea has the potential to become a global leader in personalized foods, but to achieve this, food scientists, medical professionals, and enthropologists need to work together to convince the Korean Government to lead the industry in the right direction.

\section{Author's contribution}

DYK is an author for this article. All authors read and approved the final manuscript.

\section{Competing interests}

The author declares that they have no competing interests.

Published online: 26 March 2020

References

1. What is Al? (https://aws.amazon.com/ko/machine-learning) Amazon Web Services.

2. Schwab K. The 4th Industrial Revolution (Translated by Song GJ). Seoul: Saerounhyongjae; 2016

3. Rifkin J. The Third Industrial Revolution. Seoul: Mineumsa; 2012.

4. Kim HW, Cho YJ, Gil H. Effect of Hormesis in Food. Food Eng Prog. 2014;18: 265-75

5. Davos Forum 2016. Mastering the 4th IR, Jang PS, Science and Technology Policy no. 211. Seoul: Science and technology policy institute; 2016.

6. Kwon DY. Humanities of Korean Diet. Seoul: Health letter; 2019.

7. Kwon DY. Food Industry for Sustainable Growth. Seoul: Oisikgyeongje; 2017

8. Kwon DY. Lifestyle and health in the post-industrial era. Annals Nutrit Food Sci. 2018:2:2021-8.

9. Cho YI. Artificial intelligence: technology trend and development, weekly technology trend, The Promotion Center of ICT. Seoul; 2016. 2. 17. p. 13-26.

10. Kwon DY, Lee YE, Kim MS, Kim SH. (lead authors) and 8 othersTells about a Korean diet. Seoul: Korea Food Research Institute; 2017.

11. This H. Molecular Gastronomy: Exploring the Science of Flavor. NY, New York: Columbia University Press; 2006.

12. The Newspaper: Hankyorye Sinmun, Which jobs will survive in the Al era?, March 24, 2016, Seoul, Korea.

13. Kwon DY, Chung KR. In: Park KY, Kwon DY, Lee KW, Park S, editors. Korean diets and their tastes, in Korean functional foods: composition, processing and health benefits. NY, New York: CRC Press; 2018. p. 23-42.

14. Kwon DY. The answer is in Korean diet, in longevity foods for centenarian Seoul: Sikanyun; 2019. p. 221-88.

15. Kwon DY. Why ethnic foods? J Ethn Foods. 2015;2:9.

16. Kwon DY. Seoul declaration of Korean diet. J Ethn Foods. 2016;3:1-4.

\section{Publisher's Note}

Springer Nature remains neutral with regard to jurisdictional claims in published maps and institutional affiliations.

Ready to submit your research? Choose BMC and benefit from:

- fast, convenient online submission

- thorough peer review by experienced researchers in your field

- rapid publication on acceptance

- support for research data, including large and complex data types

- gold Open Access which fosters wider collaboration and increased citations

- maximum visibility for your research: over $100 \mathrm{M}$ website views per year

At BMC, research is always in progress.

Learn more biomedcentral.com/submissions 